\title{
Medication Intervention for Chronic Kidney Disease Patients Transitioning from Hospital to Home: Study Design and Baseline Characteristics
}

\author{
Radica Z. Alicic ${ }^{a, b}$ Robert A. Short ${ }^{d}$ Cynthia L. Corbett ${ }^{e}$ Joshua J. Neumiller ${ }^{f}$ \\ Brian J. Gates $^{f}$ Kenn B. Daratha ${ }^{\mathrm{e}}$ Celestina Barbosa-Leiker ${ }^{\mathrm{e}}$ Sterling McPherson $^{\mathrm{e}}$ \\ Naomi S. Chaytor ${ }^{g}$ Brad P. Dieter ${ }^{a} \quad$ Stephen M. Setter ${ }^{f}$ Katherine R. Tuttle $^{a, c}$ \\ a Providence Health Care, bUniversity of Washington School of Medicine, 'Nephrology Division, Kidney Research \\ Institute, ${ }^{d}$ Washington State University, ${ }^{e}$ College of Nursing, ${ }^{\mathrm{f} C o l l e g e}$ of Pharmacy, and ${ }^{\mathrm{g}}$ Elson S. Floyd College of \\ Medicine, Spokane, Wash., USA
}

\section{Key Words}

Hospital admission · Primary diagnosis · Medication intervention - Pharmacist - Acute care utilization - Chronic kidney disease management $\cdot$ Elixhauser comorbidity measures

\begin{abstract}
Background: The hospital readmission rate in the population with chronic kidney disease (CKD) is high and strategies to reduce this risk are urgently needed. Methods: The CKDMedication Intervention Trial (CKD-MIT; www.clinicaltrials. gov; NCTO1459770) is a single-blind (investigators), randomized, clinical trial conducted at Providence Health Care in Spokane, Washington. Study participants are hospitalized patients with CKD stages 3-5 (not treated with kidney replacement therapy) and acute illness. The study intervention is a pharmacist-led, home-based, medication management intervention delivered within 7 days after hospital discharge. The primary outcome is a composite of hospital readmissions and visits to emergency departments and urgent care centers for 90 days following hospital discharge. Secondary outcomes are achievements of guideline-based targets for CKD risk factors and complications. Results: Enrollment began in February 2012 and ended in May 2015. At baseline, the age
\end{abstract}

of participants was $69 \pm 11$ years (mean \pm SD), $50 \%$ (77 of 155) were women, $83 \%$ (117 of 141 ) had hypertension and $56 \%$ (79 of 141) had diabetes. At baseline, the estimated glomerular filtration rate was $41 \pm 14 \mathrm{ml} / \mathrm{min} / 1.73 \mathrm{~m}^{2}$ and urine albumin-to-creatinine ratio was $43 \mathrm{mg} / \mathrm{g}$ (interquartile range $8-528 \mathrm{mg} / \mathrm{g}$ ). The most frequent diagnosis category for the index hospital admission was cardiovascular diseases at $34 \%$ (53 of 155), but the most common single diagnosis for admission was community-acquired acute kidney injury at 10\% (16 of 155). Conclusion: Participants in CKD-MIT are typical of acutely ill hospitalized patients with CKD. A medication management intervention after hospital discharge is under study to reduce post-hospitalization acute care utilization and to improve CKD management.

(c) 2016 S. Karger AG, Basel

\section{Introduction}

Patients with chronic kidney disease (CKD) experience high rates of hospitalization and readmission. An analysis of 676,343 adult patients admitted to hospitals in the Wash-

Stephen M. Setter - in memorium.

\section{KARGER}

E-Mail karger@karger.com

www.karger.com/ajn
C 2016 S. Karger AG, Basel

$0250-8095 / 16 / 0442-0122 \$ 39.50 / 0$
Radica Z. Alicic, MD

Providence Health Care

University of Washington School of Medicine

104 West 5th Avenue Suite 350E, Spokane, WA 99204 (USA)

E-Mail radica.alicic@ providence.org 
ington state demonstrated markedly elevated risk for both total readmissions and readmissions resulting in death, with risks increasing progressively by severity of CKD [1]. The United States Renal Disease System reported 30-day readmission rates at $24 \%$ for Medicare-covered CKD patients compared with $18 \%$ for those without CKD [2]. This readmission rate exceeds even a combined rate of both death and re-hospitalization in non-CKD patients [2].

Adverse drug events are common after hospital discharge and increase risk for readmissions [3, 4]. Already complex medication regimens in patients with CKD are almost always adjusted during acute illness and hospitalization resulting in a frequent occurrence of adverse drug reactions [5]. In the general population of hospitalized patients, interventions consisting of medication reconciliation by a pharmacist in conjunction with patient counseling have reduced rates of adverse drug reactions, serious complications and readmissions [6-9]. Unfortunately, the CKD population has not been a focus of concerted efforts to improve outcomes after hospital-related care. As such, there are no effective strategies to reduce readmissions and improve quality of care for hospitalized patients with CKD. Furthermore, incomplete characterization of the acutely ill CKD population limits development of effective interventional strategies to reduce hospital readmissions.

The CKD-Medication Intervention Trial (CKD-MIT) is designed to test the efficacy of a pharmacy-led, homebased medication management intervention on acute care utilization (hospital readmissions, emergency department and urgent care center visits) and CKD management following hospitalization. The objectives of the present report are to describe the study design and baseline characteristics of hospitalized patients with CKD who participated in this study.

\section{Methods}

\section{Study Design and Objectives}

CKD-MIT is a single-blind (investigators), randomized, controlled clinical trial conducted at Providence Health Care in Spokane, Washington. The aim of the study is to determine effects of a home-based, pharmacist-led intervention to improve medication management administered within 7 days after discharge on the primary outcome of a composite of hospital readmissions, emergency department visits and urgent care visits for 90 days following hospital discharge. Secondary outcomes are achievements of guideline-based targets for CKD risk factors and complications [10-16]. CKD-MIT was reviewed and approved by the Institutional Review Board, Spokane, and adhered to the principles of the Declaration of Helsinki. All study participants provided written, informed consent to join the study.

Hospital-to-Home Transition of Care for CKD
Study Participants and Enrollment

Patients with CKD were recruited from the inpatient units of a large, tertiary referral hospital between February 2012 and February 2015 with follow-up assessments completed between May 2012 and May 2015. Patients $>21$ years of age were eligible to be enrolled if they were hospitalized for an acute illness and had CKD stages 3 through 5 (not treated with kidney replacement therapy) identified by at least 2 measures of estimated glomerular filtration rate (eGFR) $<60 \mathrm{ml} / \mathrm{min} / 1.73 \mathrm{~m}^{2}$ for $>3$ months during the year prior to the index hospital admission. For study eligibility, eGFR was based on local laboratory reports using the Modification of Diet in Renal Disease calculation. Main exclusion criteria were the following: planned admission; kidney transplant or maintenance dialysis treatment; discharge to an extended care facility or rehabilitation unit; or medication management by a healthcare professional in the home.

\section{Randomization, Assessments and Intervention}

Study participants were randomized in a 1:1 ratio to the usual care discharge process alone or intervention plus usual care by means of a computer-generated random allocation sequence, stratified by diabetes status. Participants had 3 assessment visits: baseline within 7 days after hospital discharge and 2 follow-up assessments at 30 and 90 days after hospital discharge. The assessments consisted of health history, depressive symptom screening (patient health questionnaire-9), resource utilization review (emergency department visits, hospitalizations, dialysis treatment, home health services visits, rehabilitation/skilled nursing facility admissions), physical examination, health literacy screening (Rapid Estimate of Adult Literacy in Medicine - revised), cognitive screening (MINI-COGTM), clinical laboratory testing (complete metabolic panel, hemoglobin $(\mathrm{Hb})$, lipid profile, parathyroid hormone (PTH), phosphorus (PO4), urine albumin-to-creatinine ratio (UACR) and, for those with diabetes, HbA1c) and review of the medication list. Based on a pre-specified classification system, the primary diagnosis for hospitalization was adjudicated by 2 physician investigators (R.Z.A. and K.R.T.) in a blinded manner (online suppl. table 1; for all online suppl. material, see www.karger.com/ doi/10.1159/000447019). To ascertain the study participants' awareness of their kidney disease, they were asked if they knew of having 'kidney disease' or 'problems with their kidneys' at the time they consented to participate in the study. Healthcare provider awareness was assessed by identifying the diagnosis of kidney disease on the electronic health record including CKD, chronic kidney failure, acute kidney diseases, acute worsening of CKD or chronic kidney failure or specific kidney diseases. Comorbidity status of the study participants was quantified by calculating the Elixhauser comorbidity score using International Classification of Disease - 9th Revision, Clinical Modification (ICD9-CM) diagnostic codes $[1,17,18]$.

In the usual care group, participants received a medication list and discharge prescriptions with instructions presented by a nurse at the time of discharge from the hospital. They also received clinical education from the hospital staff using teach-back to verify understanding of 'red flag' signs and symptoms, filling new prescriptions right away and the importance of follow-up and review of their discharge medication regimen with their primary care provider. In addition to this usual discharge process, participants in the intervention group received a medication management intervention delivered by a home visit from a pharmacist within 7 days after hospital discharge. This intervention was previously validat-

Am J Nephrol 2016;44:122-129 


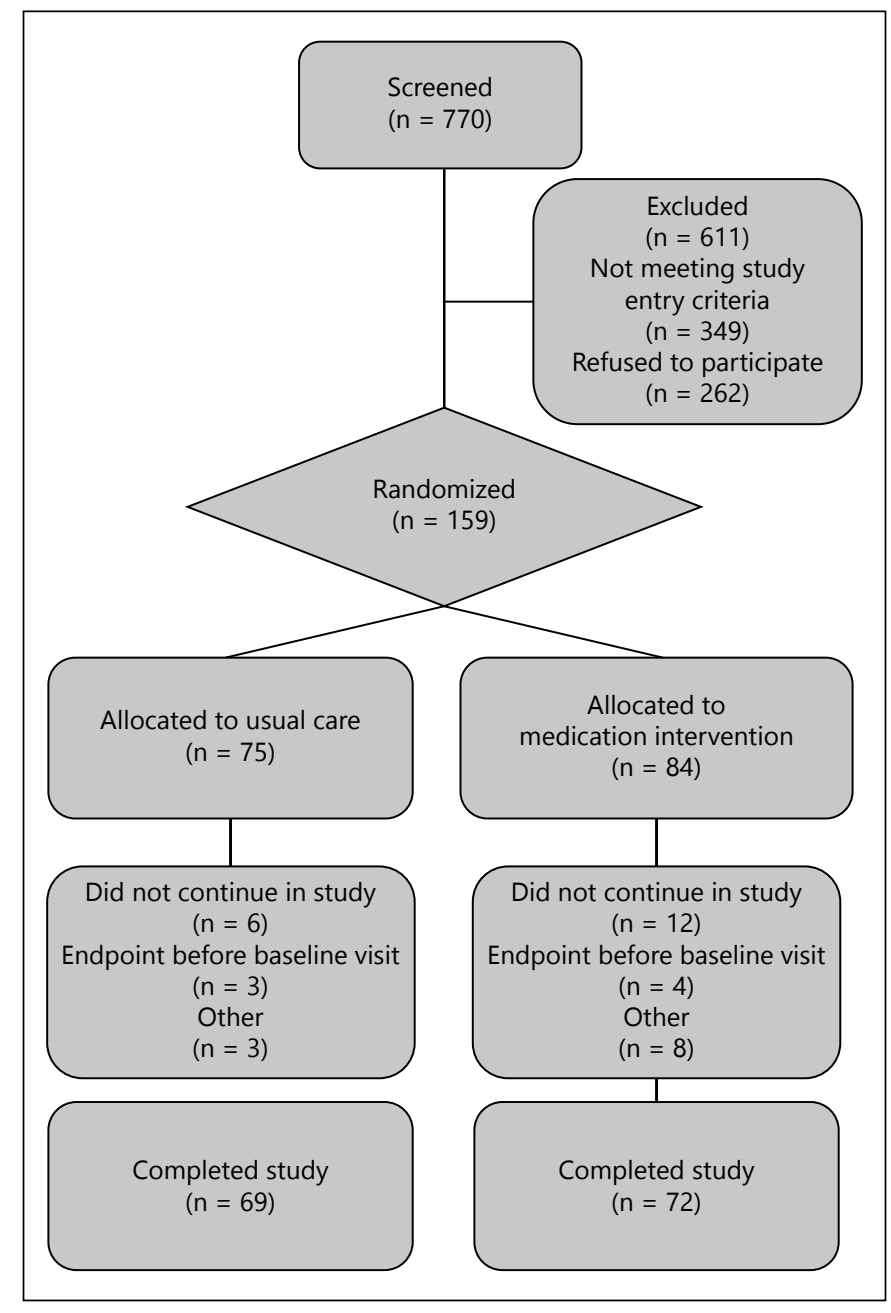

Fig. 1. CONSORT flow diagram.

ed in a general population of hospitalized patients [6]. The pharmacist-led, in-home medication management intervention is based on the Chronic Care Model and the ' $5 \mathrm{As}$ ' model of behavior change.

(1) Assessment: identify medication problems and discrepancies.

(2) Advice: answer questions or concerns, advice to carry an updated medication list, advice on medicines to avoid, resolve medication discrepancies, adjust doses for kidney function.

(3) Agreement: agree upon medications that will be taken regularly and strategies to manage the medication list and keep it updated.

(4) Assistance: develop plans for medication-taking, inform prescribers about medications that are taken differently than prescribed, create strategies to overcome barriers to keeping medication list updated.

(5) Arrangements: assistance from family or caregivers to take medicines as prescribed, keep medication list updated, obtain medications, address unresolved discrepancies, visit prescriber regarding dose adjustments or report other concerns discussed with the participant's prescriber $[19,20]$.

\section{Statistical Analyses}

A power estimate indicated that recruitment of 120 study participants would detect a $50 \%$ reduction in the primary outcome at 90 days based on a pilot study that included a subset of participants with CKD [6]. The resulting power for use of a Kaplan-Maier survival log-rank test (a 2-tailed test, and alpha of 0.05 ) was estimated at $81 \%$. Due to the loss of some enrollees prior to active study participation during the recruitment phase (CONSORT diagram; fig. 1), the sample size was subsequently increased to approximately 140 randomized participants. For baseline data analyses, eGFR was calculated from the serum creatinine concentration using the CKD epidemiology collaboration equation. Data are presented as mean \pm SD for symmetrically distributed continuous variables or median and interquartile ranges for skewed variables. Frequencies and percentages are presented with $\mathrm{n} / \mathrm{N}$ for categorical data. The comorbidity score was calculated for each participant by summing the equally weighted number of ICD9-CM diagnoses determined by Elixhauser comorbidity measures method $[1,17,18]$. In an exploratory analysis, analysis of variance and correlation coefficients were used to test the magnitude of associations between comorbidity status (Elixhauser score) and the UACR. Due to skewness, the UACR was log-transformed for analyses. All statistics were computed using the SPSS program version 22 (IBM SPSS).

\section{Results}

\section{Baseline Characteristics}

A total of 421 patients were approached to participate in the study prior to hospital discharge (CONSORT diagram; fig. 1). Of them, 262 declined to participate and 159 who consented to participate were randomly assigned to 1 of the 2 groups of the CKD-MIT trial. The most frequent reasons enrollees exited the study before the baseline visit were reaching a primary outcome (end point) event and severe illness or admission to extended care facilities $(n=18)$. Baseline demographic data and patient and provider awareness of kidney disease was available for 155 participants and a full data set was available for 141 of them.

The distribution of men and women who entered the study was nearly equal: 78 men and 77 women of a total 155 participants (table 1). Most participants, 66\% (103 of 155), were aware of their diagnosis of kidney disease. Healthcare providers noted a diagnosis of kidney disease in $79 \%$ (123 of 155) of participants. The average age of study participants was $69 \pm 11$ years. The racial distribution reflected the local community, largely of white race [21]. The baseline eGFR of the study participants was $41 \pm 14 \mathrm{ml} / \mathrm{min} / 1.73 \mathrm{~m}^{2}$ with a median UACR of $43 \mathrm{mg} / \mathrm{g}$ (interquartile range 8-528). The majority of participants had hypertension $83 \%$ (117 of 141), and 56\% (79 of 141) had diabetes. The most frequent categories of primary di- 
Table 1. Baseline characteristics of CKD-MIT participants

\begin{tabular}{|c|c|c|c|}
\hline Characteristics/attributes & $\mathrm{n} / \mathrm{N}$ & Means/median & $\% / \mathrm{SD} / \mathrm{IQR}$ \\
\hline Age, years & & 69 & 11 \\
\hline \multicolumn{4}{|l|}{ Gender } \\
\hline Women & $77 / 155$ & & 50 \\
\hline Men & $78 / 155$ & & 50 \\
\hline \multicolumn{4}{|l|}{ Race } \\
\hline White & $138 / 155$ & & 86 \\
\hline American Indian & $9 / 155$ & & 6 \\
\hline Black & $7 / 155$ & & 4 \\
\hline Others & $6 / 155$ & & 4 \\
\hline \multicolumn{4}{|l|}{ Education } \\
\hline Some high school, high school & $45 / 151$ & & 30 \\
\hline Some college, college graduate & $84 / 151$ & & 56 \\
\hline Graduate school & $22 / 151$ & & 14 \\
\hline Diabetes (yes) & $79 / 141$ & & 56 \\
\hline Hypertension (yes) & $117 / 140$ & & 83 \\
\hline $\mathrm{SBP}, \mathrm{mm} \mathrm{Hg}$ & $117 / 140$ & 131 & 21 \\
\hline Diastolic blood pressure, $\mathrm{mm} \mathrm{Hg}$ & $117 / 140$ & 73 & 11 \\
\hline eGFR (CKD-EPI), $\mathrm{ml} / \mathrm{min} / 1.73 \mathrm{~m}^{2}$ & $134 / 141$ & 41 & 14 \\
\hline CKD stage $2\left(60-89 \mathrm{ml} / \mathrm{min} / 1.73 \mathrm{~m}^{2}\right)$ & $10 / 134$ & & 8 \\
\hline CKD stage $3 \mathrm{~A}\left(45-59 \mathrm{ml} / \mathrm{min} / 1.73 \mathrm{~m}^{2}\right)$ & $38 / 134$ & & 28 \\
\hline CKD stage $3 B\left(15-29 \mathrm{ml} / \mathrm{min} / 1.73 \mathrm{~m}^{2}\right)$ & $54 / 134$ & & 40 \\
\hline CKD stage $4\left(15-29 \mathrm{ml} / \mathrm{min} / 1.73 \mathrm{~m}^{2}\right)$ & $29 / 134$ & & 22 \\
\hline CKD stage $5\left(<15 \mathrm{ml} / \mathrm{min} / 1.73 \mathrm{~m}^{2}\right)$ & $3 / 134$ & & 2 \\
\hline $\mathrm{UACR}, \mathrm{mg} / \mathrm{g}$ & $106 / 141$ & 43 & 8,528 \\
\hline Normal albuminuria $(<30 \mathrm{mg} / \mathrm{g})$ & $45 / 106$ & & 42 \\
\hline Moderately increased albuminuria (30-300 mg/g) & $29 / 106$ & & 27 \\
\hline Severely increased albuminuria $(\geq 300 \mathrm{mg} / \mathrm{g})$ & $32 / 106$ & & 30 \\
\hline Potassium, $\mathrm{mEq} / \mathrm{l}$ & $134 / 141$ & 4.6 & 0.5 \\
\hline Blood urea nitrogen, $\mathrm{mg} / \mathrm{dl}$ & $134 / 141$ & 37 & 21 \\
\hline Creatinine, $\mathrm{mg} / \mathrm{dl}$ & $134 / 141$ & 1.7 & 0.7 \\
\hline Hemoglobin, g/dl & $133 / 141$ & 11.6 & 1.9 \\
\hline $\mathrm{PTH}, \mathrm{pg} / \mathrm{ml}$ & $127 / 141$ & 76 & 62 \\
\hline $\mathrm{PO} 4, \mathrm{mg} / \mathrm{dl}$ & $132 / 141$ & 3.6 & 0.7 \\
\hline HbA1c (diabetic subgroup), \% & $77 / 141$ & 7.0 & 1.3 \\
\hline Fasting glucose (diabetic subgroup), mg/dl & $78 / 141$ & 157 & 77 \\
\hline
\end{tabular}

agnoses for hospitalization were cardiovascular disease $34 \%$ (53 of 155), infections 19\% (29 of 155) and kidney diseases $13 \%$ ( 21 of 155 ; table 2 ). The single most frequent primary diagnosis for hospitalization was acute kidney injury (AKI) $10 \%$ (16 of 155).

The study participants had $2.0 \pm 1.6$ (mean \pm SD) hospitalizations within 12 months before the index hospitalization and an Elixhauser comorbidity score of $4.6 \pm 2.5$ (mean \pm SD; online suppl. table 2). A linear relationship was observed between severity of albuminuria status and the Elixhauser comorbidity score (fig. 2). In addition, a correlation $(\mathrm{r}=0.23 ; \mathrm{p}=0.02)$ was found between the continuous variable of the log of ACR at baseline and the number of comorbidities measured by the Elixhauser method.

Hospital-to-Home Transition of Care for CKD
Frequency of use of angiotensin II receptor blocker (ARB) agents and renin angiotensin converting enzymes (ACE) inhibitors was 47\% (66 of 141; fig. 3). Of all participants, $69 \%$ (97 of 141) met the clinical goal of systolic blood pressure (SBP) $<140 \mathrm{~mm} \mathrm{Hg}$, and $51 \%$ (72 of 141) met the goal of SBP of $<130 \mathrm{~mm} \mathrm{Hg}$. Goals of diastolic blood pressure $<90$ or $<80 \mathrm{~mm} \mathrm{Hg}$ was achieved in most participants, 92\% (130 of 141) and $74 \%$ ( 104 of 141 ), respectively. $\mathrm{Hb}$ was $>11 \mathrm{~g} / \mathrm{dl}$ in $59 \%$ (79 of 133). Almost all, 98\% (129 of 132), had PO4 <5 $\mathrm{mg} / \mathrm{dl}$ and $56 \%$ (71 of 127 ) had PTH $<65 \mathrm{pg} / \mathrm{ml}$. HbAlc was $<7 \%$ in $53 \%$ ( 41 of 77 ) and $<7.5 \%$ in $69 \%$ (53 of 77 ) of study participants with diabetes. Goals were based on current clinical practice guidelines at the time of study [10-16]. 
Table 2. Adjudicated primary diagnosis for index hospitalization

\begin{tabular}{|c|c|c|}
\hline Diagnosis category (by system) & $\mathrm{n} / \mathrm{N}(\mathrm{n}=155)$ & $\%$ \\
\hline Cardiovascular diseases & $53 / 155$ & 34 \\
\hline Heart failure & $13 / 155$ & 8 \\
\hline Ischemic heart disease & $13 / 155$ & 8 \\
\hline Arrhythmias & $8 / 155$ & 6 \\
\hline Hypertension & $7 / 155$ & 4 \\
\hline Hypotension & $2 / 155$ & 1 \\
\hline Syncope & $2 / 155$ & 1 \\
\hline Pericarditis & $1 / 155$ & 1 \\
\hline Valvular abnormalities & $3 / 155$ & 2 \\
\hline Abdominal aortic aneurism & $2 / 155$ & 0.6 \\
\hline Peripheral vascular disease & $2 / 155$ & 0.6 \\
\hline Infections & $29 / 155$ & 19 \\
\hline Soft tissue/bone/joint & $9 / 155$ & 6 \\
\hline Urinary tract infection & $6 / 155$ & 4 \\
\hline Pneumonia & $7 / 155$ & 4 \\
\hline Gastrointestinal infections & $5 / 155$ & 3 \\
\hline Sepsis & $1 / 155$ & 1 \\
\hline Acute cholecystitis & $1 / 155$ & 1 \\
\hline Kidney system diseases & $21 / 155$ & 13 \\
\hline AKI & $16 / 155$ & 10 \\
\hline Nephrotic syndrome & $2 / 155$ & 1 \\
\hline Volume depletion & $3 / 155$ & 2 \\
\hline Central nervous system diseases & $12 / 155$ & 8 \\
\hline Stroke & $4 / 155$ & 3 \\
\hline Encephalopathy & $4 / 155$ & 3 \\
\hline Seizures & $1 / 155$ & 0.6 \\
\hline Intracerebral hemorrhages & $1 / 155$ & 0.6 \\
\hline Transient ischemic attack & $2 / 155$ & 1 \\
\hline Respiratory system diseases & $11 / 155$ & 7 \\
\hline Respiratory failure & $5 / 155$ & 3 \\
\hline Pleural effusion & $2 / 155$ & 1 \\
\hline Pulmonary embolism & $3 / 155$ & 2 \\
\hline Acute asthma exacerbation & $1 / 155$ & 0.6 \\
\hline Gastrointestinal system diagnosis & $10 / 155$ & 6 \\
\hline Bleeding from gastrointestinal tract & $7 / 155$ & 4 \\
\hline Cirrhosis & $1 / 155$ & 0.6 \\
\hline Small bowel obstruction & $2 / 155$ & 1 \\
\hline Miscellaneous & $19 / 155$ & 12 \\
\hline Atypical chest pain & $5 / 155$ & 3 \\
\hline Musculoskeletal & $5 / 155$ & 3 \\
\hline Malignancies & $3 / 155$ & 2 \\
\hline Hypoglycemia & $2 / 155$ & 1 \\
\hline Vasculitis & $1 / 155$ & 0.6 \\
\hline Epistaxis & $1 / 155$ & 0.6 \\
\hline Deep venous thrombosis & $1 / 155$ & 0.6 \\
\hline Revision of fistula & $1 / 155$ & 0.6 \\
\hline
\end{tabular}

\section{Discussion}

The CKD-MIT clinical trial is designed to test the efficacy of a pharmacist-led, home-delivered medication management intervention on acute care utilization and CKD care in patients recently discharged from hospital-

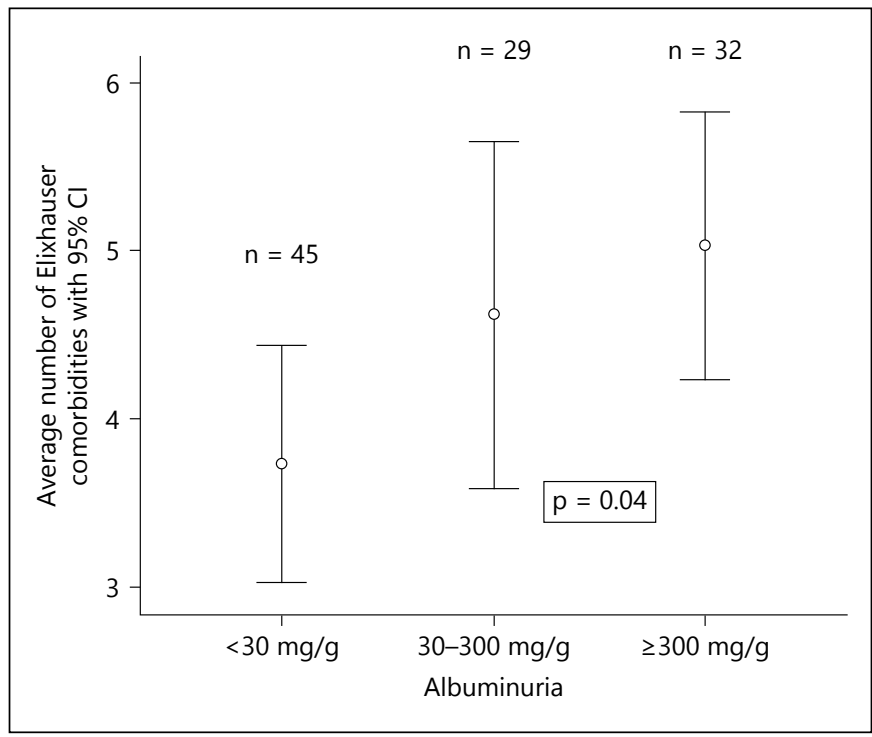

Fig. 2. Average number of Elixhauser comorbidities (with the $95 \%$ CIs) by albuminuria category $(<30,30-300, \geq 300 \mathrm{mg} / \mathrm{g})$. The probability level refers to the linear component of the association by analysis of variance.

to-home after acute illness. One of the many challenges the CKD population faces during and after hospitalization are fluctuations in kidney function related to acute illness. Declining eGFR, in particular, adds additional complexity to medication management and warrants focused attention to avert unintended consequences and complications. In the general population of hospitalized patients, tremendous clinical and administrative resources have been directed toward reducing readmission rates while improving quality of care and controlling costs. The main emphasis has been placed on sentinel conditions such as pneumonia, heart failure and myocardial infarction [22]. Although patients with CKD may have been inadvertently included in such studies, to our knowledge, this will be the first prospective, randomized, controlled clinical trial to focus an intervention on this particularly high-risk group.

The participants enrolled in CKD-MIT are older, mostly white and equally distributed between women and men. Their mean eGFR at baseline was $41 \mathrm{ml} / \mathrm{min} / 1.73 \mathrm{~m}^{2}$ and most had CKD stages $3 \mathrm{~b}-5$. As to be expected, hypertension and diabetes are very common in the study participants. In contrast to low rates of CKD awareness in observational studies, awareness of kidney disease by CKD-MIT participants and their healthcare providers was relatively high, suggesting that those who are more likely to enroll in clinical trials are more aware of their 


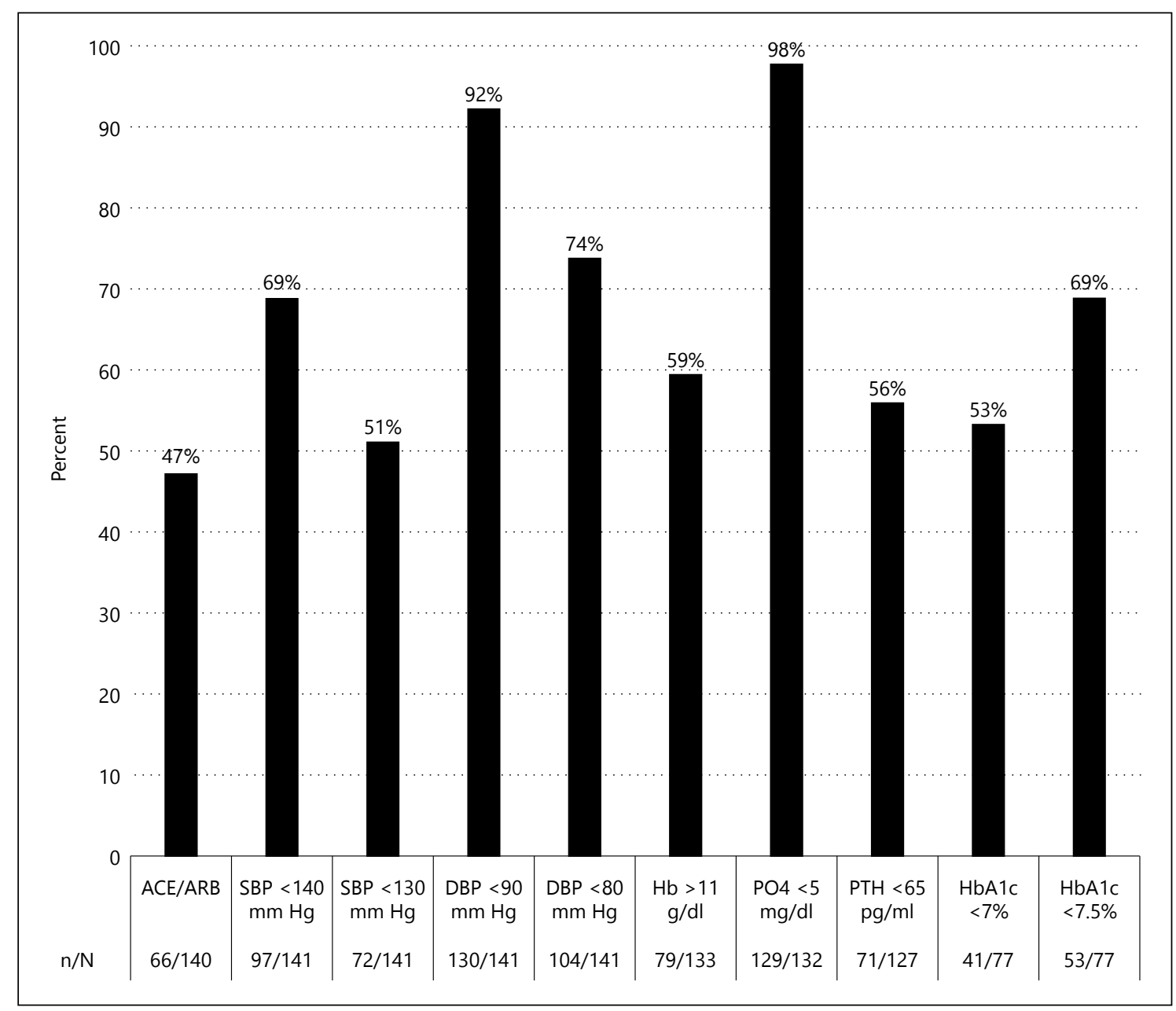

Fig. 3. Frequency of study participants achieving goals for CKD risk factor control, complications and use of ACE inhibitors and ARB medications at baseline. DBP = Diastolic blood pressure.

condition [23]. The most frequent primary diagnostic categories for hospital admission were cardiovascular diseases followed by infections and kidney diseases. Community-acquired AKI was the most common single primary diagnosis for hospitalization. Patients with CKD are known to be at higher risk of AKI [24, 25]; however, identification of community-acquired AKI as the most common primary diagnosis for admission in hospitalized patients with $\mathrm{CKD}$ is a unique finding that was captured by classification with an adjudication system rather than use of only administrative coding data.

At hospital discharge, most participants were at recommended blood pressure targets for the less stringent goal and more than half met the more intensified goal. ACE inhibitor or ARB use was less than 50\%, but these medicines are commonly stopped during acute illness and hospitalization. Recommended goals for PTH and
$\mathrm{Hb}$ were reached in more than half of participants despite acute illness and nearly all had adequate control of serum PO4 levels. HbA1c levels in patients with diabetes and $\mathrm{CKD}$ indicated acceptable glycemic control in the majority, but are more reflective of the previous 3 months of glycemic control rather than their levels of glycemia during acute illness.

In the general population, proven strategies to reduce hospital readmission rates and improve transition of care remain to be identified and validated [26-28]. A systemic review and meta-analysis of 42 trials testing interventions designed to prevent 30-day hospital readmissions found that studies published before the year 2002 were more likely to report positive results than those reported later [28]. Comprehensive interventions involving multidisciplinary care and support for self-management may be more effective than single interventions and pharmacist- 
led interventions show particular promise [7-9]. Most previously tested medication interventions took place inhospital or were conducted by a telephone call after hospital discharge. A recent study that evaluated effects of an in-person, pharmacist-led intervention performed soon after discharge in a general population of hospitalized patients showed improvements in medication management and reduced rates of acute care utilization within 30 days [3]. Additionally, a collaborative pharmacist-nurse medication intervention performed through home health services in older adults produced significant reductions in medication discrepancies [29]. CKD-MIT will provide new knowledge about the efficacy of an in-person, pharmacist-led intervention performed soon after hospital discharge on subsequent acute care utilization and CKD care.

This study population has multiple comorbidities as determined by the Elixhauser score. Of note, there was a significant relationship between severity of albuminuria and number of comorbidities, which is a novel finding from this study of patients with CKD. Similarly, in a recent study, kidney transplant recipients were found to have multiple comorbidities by the Elixhauser score [18]. Albuminuria is a well-known biomarker for kidney disease, cardiovascular disease and mortality that adds to conventional risk prediction in diabetic and non-diabetic populations alike [30, 31]. The larger implications of the relationship between severity of albuminuria and the Elixhauser comorbidity score will be further evaluated in a longitudinal manner in the CKD-MIT clinical trial.

The study design for CKD-MIT has many strengths, but also some limitations. First, focusing on the CKD population is considered as strength because of the population's high risk for post-hospitalization complications and readmissions [1]. Second, this study offers tremendous opportunity to better characterize the hospitalized population with CKD. For example, the high frequency of community-acquired AKI as a primary cause for hospital admission points toward focus on AKI prevention among patients with CKD to avoid acute illness requiring hospitalization. Third, most research in this area has been dominated by studies of administrative claims data, whereas CKD-MIT study has an abundance of direct observations and adjudicated events that allows greater delineation and knowledge about the hospitalized population with CKD. Fourth, the medication intervention is based on direct interaction with a pharmacist in the home after discharge from the hospital. Face-to-face interactions in the patient's home environment are likely to be more comprehensive than a simple phone call conducted from a distance. Pharmacists have the opportunity to review not only medication lists, but also the actual medication available in the home and observe the way patients self-administer their medicines. The pharmacist will have an opportunity to address multiple stressors that patients encounter after the hospital stay (e.g., disruption of sleeping and eating, changes in daily schedule, understanding diagnoses and health conditions). Finally, longitudinal follow-up in CKD-MIT will provide insight into medication management as well as CKD care over the 90 days after hospitalization. Limitations of the study include that it is a single-center trial, enrolling a predominantly older, well-educated, white population in the Northwestern United States. The high rate of kidney disease awareness among CKD-MIT participants and providers likely reflects selection bias for study enrollment, which is inherent to clinical trial populations and contrasts with lower reported rates of awareness in observational studies [23]. Therefore, large more diverse groups of hospitalized patients with CKD should eventually be studied to broaden generalizability of the findings.

In conclusion, the CKD-MIT is a prospective, randomized, controlled clinical trial evaluating the effect of a pharmacist-led, home-based medication management intervention during the transition of care from hospital-tohome for patients with CKD. This study will determine whether the medication management intervention reduces subsequent acute care utilization and improves CKD management. The baseline findings of CKD-MIT reveal novel findings about the hospitalized CKD population, including a high prevalence of community-acquired AKI and an association between severity of albuminuria and multiple comorbidities. The study results will be relevant to advanced care of acutely ill hospitalized patients with CKD.

\section{Acknowledgments}

The study investigators extend sincere gratitude to the dedicated study participants who volunteered for the CKD-MIT study, the study coordinators (Ruthie Franks, RN, CCRC, Kristen Frank, BS and Chris Weber, BS) for their tireless work on this project and Ms. Charlotte Mola and Joy Durham for administrative support.

\section{Disclosure Statement}

This study was supported by National Institute for Diabetes and Digestive and Kidney Diseases, Grant number R34DK094016. The funding source did not participate in the design, conduct or analysis of the study or in the decision to submit the manuscript for publication. 


\section{References}

1 Daratha KB, Short RA, Corbett CF, et al: Risks of subsequent hospitalization and death in patients with kidney disease. Clin J Am Soc Nephrol 2012;7:409-416.

2 US Renal Data System: USRDS Annual Data Report: Atlas of Chronic Kidney Disease in the United States. Bethesda, National Institute of Diabetes and Kidney Disease, 2013.

3 Hawes EM, White SF, et al: Impact of an outpatient pharmacist intervention on medication discrepancies and health care resource utilization in posthospitalization care transitions. J Prim Care Community Health 2014;5: 14-18.

4 Forster AJ, Murff HJ, Peterson JF, et al: The incidence and severity of adverse events affecting patients after discharge from the hospital. Ann Intern Med 2003;138:161-167.

5 Doody HK, Peterson GM, Watson D, et al: Retrospective evaluation of potentially inappropriate prescribing in hospitalized patients with renal impairment. Curr Med Res Opin 2015;31:525-535.

6 Corbett CF, Setter SM, Daratha KB, et al: Nurse identified hospital to home medication discrepancies: Implications for improving transitional care. Geriatr Nurs 2010;31:188196.

7 Schnipper JL, Kirwin JL, Cotugno MC, et al: Role of pharmacist counseling in preventing adverse drug events after hospitalization. Arch Intern Med 2006; 166:565-571.

8 Rennke S, Nguyen OK, Shoeb MH, et al: Hospital-initiated transitional care interventions as a patient safety strategy: a systematic review. Ann Intern Med 2013;158:433-440.

9 Gillespie U, Alassaad A, Henrohn D, et al: A comprehensive pharmacist intervention to reduce morbidity in patients 80 years or older: a randomized controlled trial. Arch Intern Med 2009;169:894-900.

10 The National Kidney Foundation-Kidney Disease Outcomes Quality Initiative (NKFKDIGO). http://www.Kidney.org/professionals/ KDOQI (accessed December 1, 2015).

11 Kidney Disease Improving Global Outcomes (KDIGO). http://www.kdigo.org (accessed December 1, 2015).
12 National Kidney Disease Education Program (NKDEP). http://nkdep.nih.gov/ professionals/clinicalpracticeguidelines.htm (accessed December 1, 2015).

13 National Diabetes Education Program (NDEP). http://www.ndep.nih.gov (accessed December 1, 2015).

14 National Kidney Foundation - Kidney Disease Outcomes Quality Initiative (NKFKDOQI). Clinical practice guidelines on hypertension and antihypertensive agents in chronic kidney disease. http://www.kidney. org/professionals/KDOQI/guidelines_bp/ index.htn (accessed December 1, 2015).

15 National Kidney Foundation - Kidney Disease Outcomes Quality Initiative (NKFKDOQI). Clinical Practice Guidelines for Diabetes and CKD. http://www.kidney.org/ professionals/KDOQI/guidelines_bp/index. htn (accessed December 1, 2015).

16 Chobanian AV, Bakris GL, Black HR, et al; National Heart, Lung, and Blood Institute Joint National Committee on Prevention, Detection, Evaluation, and Treatment of High Blood Pressure; National High Blood Pressure Education Program Coordinating Committee: The seventh report of the joint national committee on prevention, detection, evaluation, and treatment of high blood pressure: the JNC 7 report. JAMA 2003;289: 2560-2572.

17 Elixhauser A, Steiner C, Harris DR, Coffey RM: Comorbidity measures for use with administrative data. Med Care 1998;36:8-27.

18 Fabbian F, De Giorgi A, et al: Impact of comorbidity on outcome in kidney transplant recipients: a retrospective study in Italy. Intern Emerg Med 2016, Epub ahead of print.

19 Glasgow RE, Emont S, Miller DC: Assessing delivery of the five 'As' for patient-centered counseling. Health Promot Int 2006;21:245255.

20 Hung DY, Shelley DR: Multilevel analysis of the chronic care model and 5A services for treating tobacco use in urban primary care clinics. Health Serv Res 2009;44:103-127.

21 www.census.gov (accessed November 29, 2015).
22 Jha AK, Joynt KE, Orav EJ, et al: The longterm effect of premier pay for performance on patient outcomes. N Engl J Med 2012;366: 1606-1615.

23 Tuot DS, Plantinga LC, Hsu CY, et al; Centers for Disease Control Chronic Kidney Disease Surveillance Team: Chronic kidney disease awareness among individuals with clinical markers of kidney dysfunction. Clin J Am Soc Nephrol 2011;6:1838-1844.

24 James MT, de Jong P, Gansevoort RT, et al: A meta-analysis of the association of estimated GFR, albuminuria, diabetes mellitus, and hypertension with acute kidney injury. Am J Kidney Dis 2015;66:602-612.

25 Sud M, Tangri N, Pintilie M, et al: Progression to Stage 4 chronic kidney disease and death, acute kidney injury and hospitalization risk: a retrospective cohort study. Nephrol Dial Transplant 2015;pii:gfv389.

26 Hansen LO, Young RS, Hinami K, et al: Interventions to reduce 30-day rehospitalization: a systematic review. Ann Intern Med 2011;155: 520-528.

27 Shepperd S, McClaran J, Phillips CO, et al: Discharge planning from hospital to home. Cochrane Database Syst Rev 2010; 1: CD000313.

28 Leppin AL, Gionfriddo MR, Kessler M, et al: Preventing 30-day hospital readmissions: a systematic review and meta-analysis of randomized trials. JAMA Intern Med 2014;174: 1095-1107.

29 Setter SM, Corbett CF, Neumiller JJ, et al: Effectiveness of a pharmacist-nurse intervention on resolving medication discrepancies for patients transitioning from hospital to home health care. Am J Health Syst Pharm 2009;66:2027-2031.

30 Wang TJ, Evans JC, Meigs JB, et al: Low-grade albuminuria and the risks of hypertension and blood pressure progression. Circulation 2005;111:1370-1376.

31 Adler AI, Stevens RJ, Manley SE, et al; UKPDS group: Development and progression of nephropathy in type 2 diabetes: the United Kingdom prospective diabetes study (UKPDS 64). Kidney Int 2003;63:225-232. 